\title{
Prescripción de medicamentos a pacientes ambulatorios
}

\section{Prescribing drugs to patients receiving out-patient care}

\author{
F. J. Garjón
}

\section{RESUMEN}

La prescripción de medicamentos ha evolucionado para tratar principalmente patologías crónicas. Actualmente se prescribe de forma repetida usando medios informáticos, lo que acarrea problemas si no se hace con un control apropiado. Los pasos que se han propuesto para una prescripción adecuada son: definir el problema, especificar el objetivo, seleccionar el medicamento, iniciar el tratamiento cumplimentando los datos apropiados, proporcionar información, evaluar regularmente, considerar el coste y usar herramientas para reducir los errores.

Las recomendaciones para la prescripción publicadas se han centrado en pacientes ancianos e incluyen: evitar la polimedicación, revisar regularmente el tratamiento, suspender cualquier fármaco no indicado y prescribir los que el paciente precise, evitar fármacos con efectos deletéreos, usar dosis adecuadas para la edad y función renal, usar regímenes sencillos y sistemas de administración adecuados, considerar los tratamientos no farmacológicos, limitar el número de profesionales que prescriben a un paciente y evitar tratar el efecto adverso de un medicamento con otro. Se proporcionan ejemplos, extraídos del sistema de información de recetas, sobre el cumplimiento de tales recomendaciones en el Servicio Navarro de Salud-Osasunbidea. Los medios para mejorar la prescripción son: formación, auditorías, colaboración entre profesionales sanitarios y uso de herramientas informáticas.

Palabras clave. Prescripción de medicamentos. Revisión de la utilización de medicamentos. Polifarmacia. Asistencia ambulatoria.

\begin{abstract}
Drug prescription has evolved to deal mainly with chronic diseases. Nowadays, repeating prescriptions using computers results in problems if this is not done with adequate control. Steps proposed for appropriate prescription are: defining the problem; specifying the objective; selecting the drug; initiating therapy with appropriate details; giving information; regular evaluation; considering cost; and using tools to reduce errors.

Published recommendations for prescription, which have focused on elderly patients, include: avoiding polypharmacy; carrying out a regular medication review; stopping any current drugs that are not indicated and prescribing new drugs that have a clear indication; avoiding drugs that have deleterious effects; using dosages that are suitable for the age and renal function; using simple drug regimes and appropriate administration systems; considering non-pharmacological treatments; limiting the number of practitioners prescribing for each patient; and avoiding treating adverse drug reactions with further drugs. Examples of compliance with those recommendations in the Navarre Health Service, extracted from the prescription information system, are provided. The measures for improving prescription are: education, auditing, collaboration between health professionals and use of electronic tools.
\end{abstract}

Key words. Drug prescriptions. Drug utilization review. Polypharmacy. Ambulatory care.

\section{Servicio de Prestaciones Farmacéuticas. Servicio Navarro de Salud}

Recepción: 3 de febrero de 2009

Aceptación provisional: 3 de marzo de 2009

Aceptación definitiva: 10 de marzo de 2009

\section{Correspondencia:}

Francisco Javier Garjón Parra

Servicio de Prestaciones Farmacéuticas

Dirección de Atención Primaria

Servicio Navarro de Salud-Osasunbidea

Plaza de la Paz, s/n

31002 Pamplona

Tfno. 848429052

Correo electrónico: jgarjonp@navarra.es 


\section{INTRODUCCIÓN}

Jeffrey K. Aronson, presidente del comité editorial del British Journal of Clinical Pharmacology, ha definido la prescripción como "una orden escrita que incluye instrucciones detalladas de qué medicamento debe darse, a quién, en qué formulación y dosis, por qué vía, cuándo, con qué frecuencia y por cuánto tiempo. Inicia un experimento en el cual el prescriptor discute el tratamiento con el paciente e investiga y monitoriza los efectos del fármaco prescrito, con el propósito de trazar un régimen posológico que maximice los efectos beneficiosos

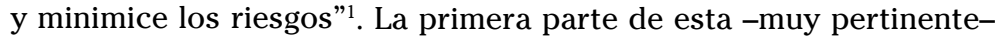
definición, ya está íntegramente recogida en nuestra legislación sobre cuáles son los datos que se deben consignar obligatoriamente en una receta médica ${ }^{2}$. La segunda hace un oportuno énfasis en considerar la prescripción no como el final sino como el comienzo de un proceso, además de señalar la incertidumbre que acompaña al acto de instaurar un tratamiento.

Es indudable que los medicamentos han aportado enormes beneficios para la salud de la población, pero también que han venido acompañados de una importante yatrogenia e ineficiencias en el uso de recursos. Se ha estimado una incidencia de efectos adversos prevenibles de medicamentos en el medio ambulatorio de 5,6 por 1.000 personas-mes ${ }^{3}$. Por incierta que sea esta cifra en nuestro ámbito, está claro que estamos ante un problema de salud de capital importancia.

El escenario de la prescripción ha evolucionado substancialmente, como lo ha hecho la práctica médica. Hemos pasado de una prescripción dirigida a problemas agudos, escrita manualmente, cara a cara con el paciente, a una prescripción repetida, generada por sistemas informáticos, a menudo separada de la visita médica. Este cambio ha sido necesario para dar respuesta a las necesidades de una población envejecida, con un enfoque más centrado en la prevención y en la atención a la patología crónica. Pero también han influido la medicalización de problemas que no son patológicos y la creación de enfermedades para ampliar el mercado de la industria farmacéutica ${ }^{4}$.

Se han identificado problemas que pueden ser agravados por la ausencia de un adecuado control clínico y administrativo de las prescripciones repetidas:

- Prescripciones incorrectas: fármacos inadecuados, polifarmacia innecesaria, no prescribir fármacos necesarios, duración demasiado corta o demasiado larga, desatender la seguridad de los medicamentos.

- Uso incorrecto por parte del paciente: incumplimiento del régimen, abandono del tratamiento sin consultar al médico.

- Mejorable relación coste-efectividad: despilfarro por usar fármacos más caros que los necesarios, continuar prescribiendo cuando el paciente no toma o no necesita la medicación o usar dosis mayores que las necesarias.

Otro factor que complica el panorama es la prescripción inducida, que obliga a un médico a recetar fármacos con los que no está familiarizado. Además puede suponer una peligrosa difusión de responsabilidades, por cuanto el que firma la receta no se siente responsable de lo prescrito. 
Adaptando las directrices de la OMS a un entorno con medios informáticos, se han propuesto ocho pasos para una prescripción adecuada $^{6}$ :

1. Evaluar y definir claramente el problema del paciente.

2. Especificar el objetivo terapéutico.

3. Seleccionar el medicamento adecuado.

4. Iniciar el tratamiento cumplimentando los datos apropiados y detallados, considerando las terapias no farmacológicas.

5. Proporcionar información, instrucciones y advertencias.

6. Evaluar regularmente el tratamiento.

7. Considerar el coste de los medicamentos al prescribir.

8. Usar los ordenadores y otras herramientas para reducir los errores de prescripción.

Los instrumentos de medición de la adecuación de la prescripción como los criterios de Beers, de McLeod, IPET (Improved Prescribing in the Elderly Tool), ACOVE (Assessing Care Of the Vulnerable Elder) o MAI (Medication Appropriateness Index) se han desarrollado para los pacientes mayores ya que debido al mayor número de fármacos consumidos y a su mayor vulnerabilidad son los más susceptibles de sufrir problemas relacionados con la medicación. Los hay explícitos como los de Beers, que generalmente se refieren a fármacos a evitar en pacientes ancianos y por lo tanto no requieren mucho juicio clínico para su aplicación. Hay que tener en cuenta que puede ser problemático el transponer directamente los criterios desarrollados para otro país. Otros son implícitos, se refieren más al paciente que al fármaco, requieren juicio clínico y son menos reproducibles y generalizables. Uno de estos últimos es el MAI, que valora el décuplo sendero de una prescripción virtuosa: indicación, dosis, efectividad, instrucciones correctas, instrucciones prácticas, interacciones fármacos-fármaco, interacciones fármaco-enfermedad, duplicidades, duración y coste $\mathrm{e}^{7-9}$.

Se han publicado recomendaciones para la prescripción que se centran en los pacientes ancianos ${ }^{10,11}$, pero son aplicables a cualquier paciente con múltiples patologías. Para proporcionar algún ejemplo que pueda ser ilustrativo de la situación en Navarra respecto a la observancia de estas recomendaciones, se realizó un estudio transversal utilizando la información de la facturación de recetas médicas del Servicio Navarro de Salud-Osasunbidea (SNS-O) correspondiente al periodo de agosto a octubre de 2008. Se incluyeron tres meses para recoger toda la información de las prescripciones crónicas al ser el máximo periodo de tiempo de que disponen las farmacias para facturar las recetas tras su dispensación y el intervalo máximo en el que pueden retirar las recetas los pacientes crónicos. Salvo que se especifique otra cosa, los datos que se proporcionan corresponden a este corte temporal. El SNS-O daba cobertura farmacéutica a 598.800 personas (un $95,4 \%$ de la población) de los cuales 142.500 tenían farmacia gratuita (pensionistas). En el periodo estudiado se facturaron 2.921.483 de recetas del SNS-O. En el $86 \%$ de las mismas se identificaba el paciente en el archivo informático mediante un código encriptado. Todas las consultas de atención primaria están informatizadas, por lo que las recetas en las que se identifica el paciente se consideran representativas de los tratamientos crónicos de los usuarios del SNS-O. No se incluyen ejemplos de prescripción dentro del hospital, sabiendo que la calidad de ésta es crucial dada la complejidad del tratamiento del paciente ingresado y la repercusión que va a tener al alta. 
Se observará que las recomendaciones emitidas son de mero sentido común:

\section{Evitar la polimedicación en la medida de lo posible}

Cuantos más fármacos lleve un paciente más probables son los problemas relacionados con medicamentos. Esta obviedad es debida a una combinación de factores como son la pura probabilidad, más interacciones, las dificultades de manejo o el peor estado del paciente. Naturalmente un paciente con varias patologías puede necesitar numerosos fármacos, pero la adición de cada nuevo medicamento no debe hacerse de manera mecánica, sino valorando el conjunto del tratamiento. Se genera una polimedicación innecesaria con las duplicidades, la prescripción en cascada (tratar el efecto adverso de un medicamento con otro) y cuando se añaden fármacos por falta de respuesta por incumplimiento del tratamiento original ${ }^{8}$.

A 21.016 pacientes (el 3,6\% del total) se les estaba prescribiendo diez o más medicamentos diferentes. A 3.764 (el 0,6\%) se les prescribieron quince o más. El número máximo de principios activos distintos que ha recibido un solo paciente es de 40.

Como se ve, son numerosos los pacientes que llevan terapias de una enorme complejidad. Su control supone un formidable reto para los profesionales sanitarios, no digamos para los propios pacientes. Suponer que un anciano con problemas de los sentidos vaya a ser capaz de manejar correctamente el tratamiento simultáneo con quince medicamentos diferentes es de un optimismo quizá irresponsable.

\section{Revisar regularmente la medicación y discutir y acordar los cambios con el paciente}

Se recomienda hacerlo con cada nuevo paciente, cada seis a doce meses y cuando haya cambios en el tratamiento ${ }^{10}$.

Preguntas a hacerse durante la revisión de la medicación (cuestiones Hamdy) ${ }^{10}$ :

- ¿Está todavía presente la indicación para la que el medicamento fue originalmente prescrito?

- ¿Hay duplicaciones en la terapia farmacológica? ¿Es posible alguna simplificación?

- ¿Incluye el tratamiento fármacos prescritos para una reacción adversa? Si es así, ¿se puede retirar el fármaco original?

- ¿Puede la dosis actual ser subterapéutica o tóxica debido a la edad del paciente o a su función renal?

- ¿Hay alguna interacción significativa fármaco-fármaco o fármaco-enfermedad?

Con motivo de un reciente estudio realizado en una muestra de médicos generales de Noruega, se han definido unos criterios de calidad de la prescripción en pacientes ancianos. Varios se refieren al uso de antiinflamatorios no esteroideos (AINE) con otros fármacos cuya combinación acarrea riesgos ${ }^{12,13}$. En la tabla 1 se ve el porcentaje de pacientes mayores de 70 años del SNS-O que sufren algunas estas combinaciones y los datos del citado estudio.

Es frecuente el uso en ancianos de múltiples fármacos con acción sobre el sistema nervioso central. Se ha propuesto como indicador 
de baja calidad el número de pacientes ancianos que reciben tres o más fármacos psicotrópicos (opioides, antipsicóticos, hipnóticos, sedantes o antidepresivos $)^{13}$. En un 7,2\% de los pacientes mayores de 70 años del SNS-O se da esta circunstancia.

Tabla 1. Prevalencia de asociaciones con AINE que acarrean riesgos en ancianos.

\begin{tabular}{|l|l|c|c|}
\hline \multirow{2}{*}{ Combinación } & \multirow{2}{*}{ Riesgo asociado } & \multicolumn{2}{|c|}{ \% de los pacientes mayores de70 años } \\
\cline { 3 - 4 } & & Navarra & Noruega (IC95\%) \\
\hline $\begin{array}{l}\text { Anticoagulantes } \\
\text { orales y AINE }\end{array}$ & $\begin{array}{l}\text { Sangrado } \\
\text { gastrointestinal }\end{array}$ & $0,9 \%$ & $0,3 \%(0,3$ a 0,4$)$ \\
\hline $\begin{array}{l}\text { IECA o ARA II } \\
\text { y AINE }\end{array}$ & Fallo renal & $7,9 \%$ & $3,4 \%(3,2$ a 3,6$)$ \\
\hline $\begin{array}{l}\text { Diuréticos } \\
\text { y AINE }\end{array}$ & $\begin{array}{l}\text { Reducción } \\
\text { del efecto del } \\
\text { diurético }\end{array}$ & $7,0 \%$ & $2,4 \%(2,2$ a 2,5$)$ \\
\hline ISRS y AINE & $\begin{array}{l}\text { Sangrado } \\
\text { gastrointestinal }\end{array}$ & $1,9 \%$ & $0,8 \%(0,7$ a 0,9$)$ \\
\hline
\end{tabular}

El 8,1\% de los pacientes que recibían un inhibidor de la colinesterasa (donepezilo, galantamina, rivastigmina) estaban tomando también un fármaco con acción anticolinérgica (antidepresivos tricíclicos, antipsicóticos fenotiazídicos, antihistamínicos de primera generación o antiespasmódicos urinarios). Los efectos de estos fármacos se oponen los unos a los otros ${ }^{14,15}$. Además, los fármacos con acción anticolinérgica son ellos mismos causa de problemas cognitivos en los pacientes ancianos ${ }^{16}$.

\section{Suspender cualquier fármaco que no esté indicado y prescribir aquellos fármacos que precise el paciente}

Se ha advertido de la frecuente prescripción de los inhibidores de la bomba de protones sin una indicación clara ${ }^{17,18}$. En el SNS-O se les prescribía algún inhibidor de la bomba de protones a 67.194 pacientes, esto es el 11,0\% del total de la población.

La sobreprescripción no excluye la infraprescripción de fármacos necesarios. De los 3.954 pacientes tratados simultáneamente con ácido acetilsalicílico y AINE, el 27,0\% no llevaban gastroprotección con inhibidores de la bomba de protones o misoprostol.

\section{Si es posible, evitar los fármacos que se sabe que tienen efectos deletéreos en los pacientes ancianos, como las benzodiazepinas}

En la figura 1 se aprecia la evolución del número de dosis diarias definidas por cada mil pacientes (DHD) pensionistas en Navarra.

En las fichas técnicas de las benzodiazepinas (cuando hablamos de benzodiazepinas, incluimos los fármacos relacionados con éstas [zolpidem, zopliclona y zaleplon)] se ha incluido una advertencia dirigida a evitar la cronificación de su uso que casi parece un sarcasmo a la vista de los datos de prescripción: "La duración del tratamiento debe ser lo más corta posible. De forma general, la duración máxima 
del tratamiento será de cuatro semanas para el insomnio y de doce semanas para la ansiedad generalizada, incluyendo la retirada gradual del medicamento".

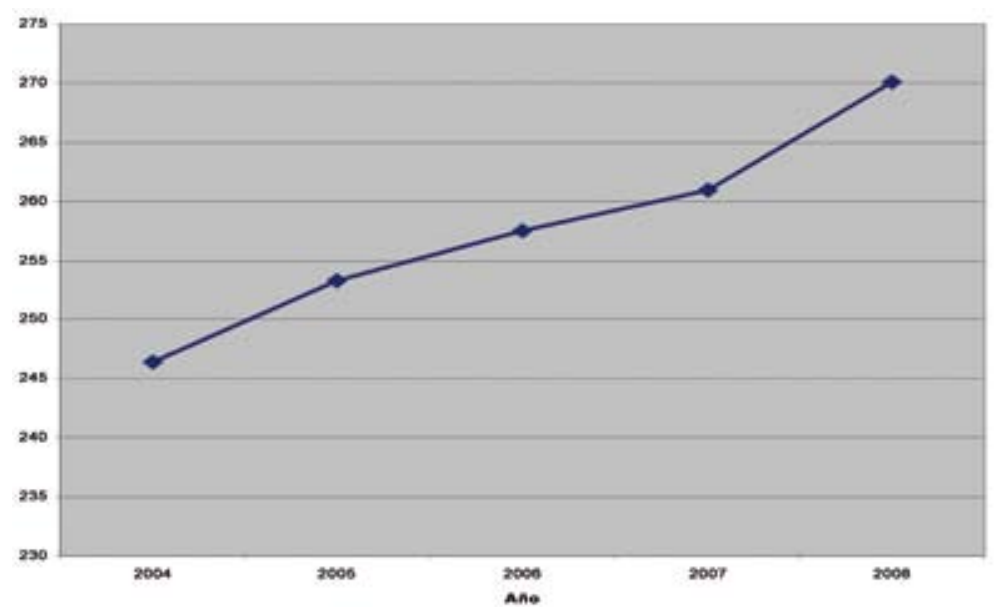

Figura 1. DHD de benzodiazepinas en pensionistas en Navarra.

De los 26.864 pacientes mayores de 65 años que recibieron una benzodiazepina (un $26,1 \%$ del total de mayores de 65 ) en el periodo agosto-octubre, el $83,9 \%$ ya las estaban utilizando en el periodo enero-marzo del 2008. Está claro que la mayoría de tratamientos se cronifican en abierta contradicción con las recomendaciones sobre el manejo de estos fármacos.

No es infrecuente el tratamiento con varias benzodiazepinas simultáneamente, algo poco justificable desde el punto de vista farmacológico. El número de pacientes que recibían tres o más benzodiazepinas diferentes fue de 739 .

\section{Usar las dosis adecuadas para la edad y la función renal del paciente}

Desde hace tiempo, en la ficha técnica de los medicamentos figura obligatoriamente la posología en ancianos, y en pacientes con insuficiencia renal o hepática. Esta información debería ser considerada en cada caso. Una baja tasa de filtrado glomerular es un predictor de problemas con los medicamentos. Su cálculo es sencillo mediante la fórmula de Cockcroft-Gault y debería utilizarse de forma habitual para el ajuste de dosis en pacientes de riesgo.

\section{Usar regímenes sencillos y sistemas de administración adecuados utilizando formulaciones de posología simple y asociaciones cuando sean apropiadas}

\section{Considerar los tratamientos no farmacológicos}

Para algunos de los problemas más frecuentes como la artrosis, el insomnio o el estreñimiento, las terapias no farmacológicas no sólo 
son de utilidad, sino que a largo plazo van a constituir la mejor forma de abordar los problemas.

\section{Limitar el número de profesionales que prescriben a un paciente}

Un estudio ha mostrado que el número de médicos prescriptores es un factor de riesgo independiente de sufrir efectos adversos de medicamentos ${ }^{19}$. Este es un campo en el que los médicos de familia no pueden hacer dejación de su función; aunque obviamente los especialistas son responsables de prescribir con calidad, conociendo todos los medicamentos que toma el paciente. Al médico de familia le corresponde ordenar los tratamientos prescritos por los diversos especialistas y los recomendados por enfermería, de la forma que mejor se ajuste a las necesidades del paciente manteniendo una visión integral de éste.

\section{En la medida de lo posible, evitar tratar el efecto adverso de un medicamento con otro fármaco}

Recientemente la Food and Drug Administration estadounidense ha lanzado una alerta, información ya recogida en las fichas técnicas, que advierte del riesgo de dolor músculo-esquelético grave y a veces incapacitante en pacientes que toman bisfosfonatos y recomienda retirar el fármaco cuando ocurra ${ }^{20}$.

De los 12.765 pacientes del SNS-O que tomaban bisfosfonatos, el $60 \%$ también estaba tratado con antiinflamatorios no esteroideos o analgésicos. Ciertamente son numerosas las indicaciones que justifican estos tratamientos, pero cabe preguntarse si el dolor causado por los bisfosfonatos ha sido considerado en muchos de estos pacientes.

\section{¿CÓMO AFRONTAR EL PROBLEMA DE LA PRESCRIPCIÓN?}

Los mismos agentes que pueden estar agravando la situación, podrían, si se utilizaran bien, contribuir a la mejora.

\section{Formación}

Una formación adecuada en prescripción resulta fundamental ${ }^{21}$. Las visitas educativas solas o combinadas con otras intervenciones tienen efectos sobre la prescripción que, aunque pequeños, pueden ser potencialmente importantes ${ }^{22}$. Lo que no tiene sentido es dejar la formación médica continuada en manos de la industria farmacéutica que es como poner a los lobos cuidando del rebaño. Esto no va a ayudar a racionalizar el uso de fármacos ${ }^{23}$.

\section{Auditorías}

Puede servir en la medida en que la información sobre la prescripción genere una retroalimentación rápida y dirigida a mejoras concretas. Es probable que la efectividad relativa de la auditoría y la retroalimentación sea mayor cuando el cumplimiento al inicio de la práctica recomendada es bajo ${ }^{11,24}$. 
En general, ninguna intervención educativa o auditora aislada tiene mucho efecto en cambiar las prácticas de prescripción, los mayores resultados pueden obtenerse con intervenciones multifacéticas tras identificar las barreras para el cambio ${ }^{25}$.

La formación e información sobre medicamentos se ven entorpecidas por la escasez de investigaciones en resultados en salud que respondan a las preguntas que importan a clínicos y pacientes y no sólo a los intereses de la industria farmacéutica.

\section{Colaboración entre profesionales sanitarios}

Una excusa que frecuentemente se aduce para no llevar a cabo revisiones periódicas de la medicación de los pacientes es la falta de tiempo en las consultas. Enfermeros y farmacéuticos pueden ser de gran ayuda en aspectos tales como controlar el cumplimiento terapéutico, vigilar la aparición de efectos adversos e informar de posibles interacciones ${ }^{26,27}$, así como en evaluar y adaptar la medicación al alta hospitalaria ${ }^{28,29}$. Todo ello sin perder de vista la función del médico de familia como responsable último del tratamiento del paciente. Diversos profesionales actuando descoordinadamente pueden hacer más mal que bien ${ }^{30,31}$.

\section{Uso de herramientas informáticas}

El tratar de informatizar el caos es un atajo hacia el desastre. Pero la aplicación juiciosa de la informática puede constituir una poderosa ayuda. Existen algunas pruebas de que la prescripción electrónica puede reducir los errores de medicación y los efectos adversos. Los estudios se han llevado a cabo fundamentalmente en el medio hospitalario y con sistemas de apoyo a la decisión clínica ${ }^{32,33}$. En este sentido se han propuesto las prestaciones que debería tener un sistema de prescripción electrónica con ayudas a la decisión clínica sobre la medicación $^{34}$ :

- Nivel básico: control de alergias a fármacos, guía básica de dosificación, ayuda de decisiones de formulario, control de duplicidades y de interacciones medicamentosas.

- Nivel avanzado: ayuda de dosificación en insuficiencia renal y en pacientes ancianos, guía de controles de laboratorio relacionados con la medicación, control de fármacos y embarazo, control de contraindicaciones fármaco-enfermedad.

Los clínicos pueden comparar lo que están ofreciendo sus sistemas de prescripción con este horizonte; siempre teniendo en cuenta que la informática, por sí sola, no va a arreglar una prescripción descuidada sino que debe acompañarse del esfuerzo del médico que persigue la mejora.

\section{CONSIDERACIONES FINALES}

Examinado sólamente datos de dispensación de recetas, se han hallado variadas muestras de prácticas de prescripción desafortunadas. Si a esta información se añadiera el diagnóstico, se podrían afinar las advertencias y detectar nuevas áreas de mejora. 
Resulta difícil exagerar la importancia de una prescripción apropiada. Sólo hay que pensar que después de una detallada anamnesis, una solicitud y cuidadosa valoración de pruebas, tras un meticuloso diagnóstico diferencial, frecuentemente el único procedimiento terapéutico que recibe el paciente es la prescripción de un fármaco. $\mathrm{Si}$ ésta es inadecuada, se puede haber echado por tierra todo el trabajo anterior.

El tratamiento del paciente no debe limitarse a añadir fármacos sobre cada problema de salud detectado. Cada prescripción debe valorase en función del resto de tratamientos del paciente y de su situación general en un proceso en continua revisión. La prescripción correcta es una responsabilidad de todos los profesionales sanitarios, pero es innegable el papel central del médico de familia en quien confluye toda la información sobre el paciente y está en situación de tener una visión holística de sus problemas.

Aunque los tratamientos farmacológicos han crecido en complejidad, también se han desarrollado los sistemas de información sobre medicamentos que pueden ayudar a su manejo. El desafío está en encontrar los medios para transferir esta información a la consulta del médico de forma que cumpla su cometido que no es otro que el beneficio del paciente.

\section{$\overline{\text { Agradecimientos }}$}

A Cristina Agudo, Maite Artázcoz, Ana Azparren, Juan Erviti, Javier Gorricho, Antonio López y Lourdes Muruzábal por sus valiosos comentarios.

\section{BIBLIOGRAFÍA}

1. ARonson JK. Balanced prescribing. Br J Clin Pharmacol 2006; 62: 629-632.

2. Ministerio de Sanidad y Consumo. Real Decreto 1910/1984 de 26 de septiembre de receta médica. (BOE n. 259 de 29/10/1984).

3. Thomsen LA, Winterstein AG, Sondergaard B, Haugbolle LS, Melander A. Systematic review of the incidence and characteristics of preventable adverse drug events in ambulatory care. Ann Pharmacother 2007; 41: 1411-1426.

4. Moynihan R, Heath I, Henry D, Gotzsche PC. Selling sickness: the pharmaceutical industry and disease mongering Commentary: Medicalisation of risk factors. BMJ 2002; 324: 886-891.

5. De Smet PA, Dautzenberg M. Repeat prescribing: scale, problems and quality management in ambulatory care patients. Drugs 2004; 64: 1779-1800.

6. Pollock M, Bazaldua OV, Dobbie AE. Appropriate prescribing of medications: an eight-step approach. Am Fam Physician 2007; 75: 231-236.

7. Spinewine A, Schmader KE, Barber N, Hughes C, Lapane KL, Swine C et al. Appropriate prescribing in elderly people: how well can it be measured and optimised? Lancet 2007; 370: 173-184.

8. Gallagher P, Barry P, O'MAHONy D. Inappropriate prescribing in the elderly. J Clin Pharm Ther 2007; 32: 113-121.

9. O'MAHONY D, GALLAGHER PF. Inappropriate prescribing in the older population: need for new criteria. Age Ageing 2008; 37: 138-141.

10. Pham CB, Dickman RL. Minimizing adverse drug events in older patients. Am Fam Physician 2007; 76: 1837-1844. 
11. Mitton JC, HiLl-Smith I, Jackson SH. Prescribing for older people. BMJ 2008; 336: 606-609.

12. Brekke M, Rognstad S, Straand Jr, Furu K, Gjelstad S, Bjørner T et al. Pharmacologically inappropriate prescriptions for elderly patients in general practice: How common? Scand J Prim Health Care 2008; 26: 80-85.

13. Straand J, Fetveit A, Rognstad S, Gjelstad S, BrekKe M, Dalen I. A cluster-randomized educational intervention to reduce inappropriate prescription patterns for elderly patients in general practice-The Prescription Peer Academic Detailing (Rx-PAD) study [NCT00281450]. BMC Health Serv Res 2006; 6: 72 .

14. Gill SS, Mamdani M, Naglie G, Streiner DL, Bronskill SE, Kopp A et al. A prescribing cascade involving cholinesterase inhibitors and anticholinergic drugs. Arch Intern Med 2005; 165: 808-813.

15. JoHNELL K, FASTBOM J. Concurrent use of anticholinergic drugs and cholinesterase inhibitors: register-based study of over 700,000 elderly patients. Drugs \& Aging 2008; 25: 871-877.

16. Hilmer SN, Mager DE, Simonsick EM, Cao Y, Ling SM, Windham BG et al. A drug burden index to define the functional burden of medications in older people. Arch Intern Med 2007; 167: 781-787.

17. Forgacs I, Loganayagam A. Overprescribing proton pump inhibitors. BMJ 2008; 336: 2-3.

18. de Burgos lunar C, Novo del Castillo S, Llorente Diaz E, Salinero Fort Ma. Estudio de prescripción-indización de inhibidores de la bomba de protones. Rev Clin Esp 2006; 206: 266-270.

19. Green JL, Hawley JN, Rask KJ. Is the number of prescribing physicians an independent risk factor for adverse drug events in an elderly outpatient population? Am J Geriatr Pharmacother 2007; 5: 31-39.

20. U.S. Drug and Food Administration. Information on Bisphosphonates (marketed as Actonel, Actonel+Ca, Aredia, Boniva, Didronel, Fosamax, Fosamax+D, Reclast, Skelid, and Zometa) 2008.

21. Aronson JK, Henderson G, WebB DJ, Rawlins MD. A prescription for better prescribing. BMJ 2006; 333: 459-460.

22. O'Brien MA, Rogers S, Jamtvedt G, Oxman AD, OdgaArd-Jensen J, Kristoffersen DT et al. Visitas de extensión educativas: efectos sobre la práctica profesional y los resultados de salud (Revisión Cochrane traducida). En: La Biblioteca Cochrane Plus, 2008 Número 4. Oxford: Update Software Ltd. Disponible en: http://www.update-software.com. (Traducida de The Cochrane Library, 2008 Issue 3. Chichester, UK: John Wiley \& Sons, Ltd.).

23. MoyniHAn R. Is the relationship between pharma and medical education on the rocks? BMJ 2008; 337: a925.

24. Jamtvedt G YJ, KRIStoffersen DT, O'Brien MA, Oxman AD. Efectos de la auditoría y la retroalimentación (feedback) en la práctica profesional y las medidas de resultado de atención sanitaria (Revisión Cochrane traducida). En: La Biblioteca Cochrane Plus, 2008 Número 4. Oxford: Update Software Ltd. Disponible en: http://www.update-software.com. (Traducida de The Cochrane Library, 2008 Issue 3. Chichester, UK: John Wiley \& Sons, Ltd.).

25. ARNOLD SR SS. Intervenciones para mejorar las prácticas de prescripción de antibióticos en la atención ambulatoria (Revisión Cochrane traducida). En: La Biblioteca Cochrane Plus, 2008 Número 4. Oxford: Update Software Ltd. Disponible en: http://www.update-software.com. (Traducida de The Cochrane Library, 2008 Issue 3. Chichester, UK: John Wiley \& Sons, Ltd.).

26. Royal S, Smeaton L, Avery AJ, Hurwitz B, SheiKh A. Interventions in primary care to reduce medication related adverse events and hospital admissions: systematic review and meta-analysis. Qual Saf Health Care 2006; 15: 23-31.

27. Wu JYF, Leung WYS, Chang S, Lee B, Zee B, Tong PCY et al. Effectiveness of telephone counselling by a pharmacist in reducing mortality in patients receiving polypharmacy: randomised controlled trial. BMJ 2006; 333: 522. 
28. Kaboli PJ, Hoth AB, McClimon BJ, Schnipper JL. Clinical pharmacists and inpatient medical care: a systematic review. Arch Intern Med 2006; 166 : 955-964.

29. MANNo MS, HAYEs DD. Best-practice interventions: how medication reconciliation saves lives. Nursing 2006; 36: 63-64.

30. Holland R, Lenaghan E, Harvey I, Smith R, Shepstone L, Lipp A et al. Does home based medication review keep older people out of hospital? The HOMER randomised controlled trial. BMJ 2005; 330: 293.

31. Salter C, Holland R, Harvey I, Henwood K. "I haven't even phoned my doctor yet." The advice giving role of the pharmacist during consultations for medication review with patients aged 80 or more: qualitative discourse analysis. BMJ 2007; 334: 1101.

32. Shamliyan TA, Dural S, Du J, Kane RL. Just what the doctor ordered. Review of the evidence of the impact of computerized physician order entry system on medication errors. Health Serv Res 2008; 43 (1pt1): 32-53.

33. Ammenwerth E, Schnell-Inderst P, Machan C, Siebert U. The effect of electronic prescribing on medication errors and adverse drug events: a systematic review. J Am Med Inform Assoc 2008; 15: 585-600.

34. Kuperman GJ, Bobb A, Payne TH, Avery AJ, Gandhi TK, Burns G et al. Medicationrelated clinical decision support in computerized provider order entry systems: a review. J Am Med Inform Assoc 2007; 14: 29-40. 
